\title{
P-Glycoprotein Overexpression Is Associated With Cisplatin Resistance in Human Osteosarcoma
}

\author{
CHAO HE ${ }^{1 *}$, ZHENYU SUN $^{1 *}$, ROBERT M. HOFFMAN ${ }^{2,3}$, ZHIJIAN YANG $^{2}$, \\ YUHANG JIANG $^{1}$, LEI WANG $^{1}$ and YONGQIANG HAO ${ }^{1}$ \\ ${ }^{1}$ Shanghai Key Laboratory of Orthopedic Implants, Department of Orthopedic Surgery, \\ Shanghai Ninth People's Hospital, Shanghai Jiao Tong University School of Medicine, Shanghai, P.R. China; \\ ${ }^{2}$ AntiCancer, Inc., San Diego, CA, U.S.A.; \\ ${ }^{3}$ Department of Surgery, University of California, San Diego, CA, U.S.A.
}

\begin{abstract}
Background/Aim: Osteosarcoma (OS) is a diagnosed primary cancer of the bone. Despite the great advances that have been made during the past decades in $O S$ therapy, drug resistance and tumor recurrence are still major problems. It is urgent to find novel strategies to overcome drug resistance in order to prolong the survival time of OS patients. Materials and Methods: Cell viability was investigated by the cell count kit-8 (CCK-8) and colony formation assays. PGlycoprotein ( $P$-gp) expression was analyzed by RT-qPCR and western blot. A xenograft mouse model was used to identify the synergistic efficacy of a P-gp inhibitor with cisplatin. Student's t-test was used to determine statistically significant differences. Results: P-gp expression levels were associated with cisplatin efficacy in OS patients. OS cells with higher P-gp expression were more resistant to cisplatin. Knockdown or inhibition of $P$ gp sensitized OS cells to cisplatin. Conclusion: Downregulating the expression of $P$-gp in $O S$ maybe a promising strategy to overcome cisplatin resistance.
\end{abstract}

This article is freely accessible online.

*These Authors contributed equally to this work.

Correspondence to: Yuhang Jiang, Shanghai Key Laboratory of Orthopedic Implants, Department of Orthopedic Surgery, Shanghai Ninth People's Hospital, Shanghai Jiao Tong University School of Medicine, Shanghai 200011, P.R. China. Tel: +86 2123271699, email: xhaka2016@163.com; Lei Wang, Shanghai Key Laboratory of Orthopedic Implants, Department of Orthopedic Surgery, Shanghai Ninth People's Hospital, Shanghai Jiao Tong University School of Medicine, Shanghai 200011, P.R. China. Tel: +86 2123271699, e-mail: wanglei12041985@163.com; and Yongqiang Hao, Shanghai Key Laboratory of Orthopedic Implants, Department of Orthopedic Surgery, Shanghai Ninth People's Hospital, Shanghai Jiao Tong University School of Medicine, Shanghai 200011, P.R. China. Tel: +86 2123271699, e-mail: haoyq1664@sh9hospital.org

Key Words: Osteosarcoma, P-Glycoprotein, cisplatin resistance.
Osteosarcoma (OS) is a rare primary cancer of the bone that mainly affects adolescents (1). The main treatment of OS is surgery and adjuvant chemotherapy. First-line chemotherapy usually includes a combination of methotrexate, doxorubicin, cisplatin, and ifosfamide. Unfortunately, chemotherapy often fails due to the appearance of multidrug resistance (MDR) (2). The prognosis of OS remains unchanged for decades. The survival time in recurrent OS patients with drug resistance is approximately one year (3-5). Hence, it is critical to overcome MDR to prolong the survival time of OS patients $(2,6-8)$. The up-regulation of the drug efflux pump P-glycoprotein (P-gp) appears critical for MDR (8-10).

$\mathrm{P}$-gp is coded by the gene $A B C B 1$ (or $M D R 1$ ) and belongs to the ATP-binding cassette $(\mathrm{ABC})$ membrane transport superfamily (11). P-gp has 12 transmembrane regions and two ATP-binding sites. This structure enables efflux of broadspectrum drugs including positively-charged hydrophobic drugs (12). The substrates of P-gp include taxanes, vinca alkaloids, podophyllotoxins and anthracyclines (13). P-gp was found up-regulated in many cancers, such as breast cancer, neuroblastoma, acute lymphocytic leukemia (ALL), acute nonlymphocytic leukemia (ANLL), neuroblastoma and pheochromocytoma (14). Overexpressed P-gp will accelerate the excretion of drugs and attenuate chemotherapy efficacy against these tumors.

In our study, expression of P-gp was found to be higher in OS patient specimens resistant to cisplatin compared to those sensitive to cisplatin. Also, knockdown or inhibition of $\mathrm{P}$-gp sensitized OS to cisplatin in vitro and in vivo. The results suggested that $\mathrm{P}$-gp is a promising target for cisplatin -resistance in OS.

\section{Materials and Methods}

Tissue samples collection. The patients involved in this study had histologically confirmed OS and they provided informed consents. The study was performed following the regulations of the ethics 
committee of the Shanghai Ninth People's Hospital. A total of 12 patients (4 cisplatin sensitive and 8 cisplatin resistant OS patients) were included.

Immunohistochemistry (IHC). IHC was produced as described (15). Briefly, after deparaffinization, rehydration, antigen retrieval and endogenous peroxidase blocking, sections were incubated with the primary antibody: anti-P-gp (Cell Signaling Technology, Danvers, MA USA) at $4{ }^{\circ} \mathrm{C}$ overnight. After rinsing, the secondary antibody was used for $20 \mathrm{~min}$ at room temperature and the sections were counterstained with hematoxylin (Sigma, St. Louis, MO, USA).

Cells. Human OS cell lines, HOS, U-2OS, Saos-2, and MG-63 cells were purchased from the Shanghai Institute of Biochemistry and Cell Biology, Shanghai, China. Cells were grown in DMEM (HyClone, Waltham, MA, USA) supplemented with 10\% FBS (Gibco, Waltham, MA, USA) and antibiotics (penicillin $100 \mathrm{U} / \mathrm{ml}$, streptomycin $100 \mu \mathrm{g} / \mathrm{ml}$ ) and $5 \% \mathrm{CO}_{2}$ at $37^{\circ} \mathrm{C}$ humidified atmosphere.

Cell viability assay. The assay was performed as described (16). In brief, cells were seeded in 96-well plates. After incubation for $72 \mathrm{~h}$ with different concentrations of cisplatin, with or without dofequidar fumarate $(5 \mu \mathrm{M})$ (MedChemExpress, Shanghai, PR China) - an inhibitor of P-gp (17-20), we added $10 \mu \mathrm{CCK}-8$ to each well and incubated at $37^{\circ} \mathrm{C}$ for $2 \mathrm{~h}$.

Colony formation assay. Cells were added in 6 -well plates at $5 \times 10^{3}$ cells per well. After cell attachment, different concentrations of cisplatin $(0 \mu \mathrm{M}, 0.4 \mu \mathrm{M}, 0.8 \mu \mathrm{M})$ with or without dofequidar fumarate $(5 \mu \mathrm{M})$ were added. After 7 days, we fixed colonies and stained them with $0.1 \%$ crystal violet.

$R N A$ extraction and $R T-q P C R$. The experiments were conducted as previously described (21), RNA was extracted by TRIzol Reagent (Takara, Shanghai, PR China). RT-qPCR was performed according to the protocols. Data were calculated as $2-\Delta \Delta \mathrm{Ct}$. The primer sequences are listed in Table I.

Western blot. An equal amount of protein was subjected to $10 \%$ dodecyl sulfate, sodium salt (SDS)-polyacrylamide gel electrophoresis (SDS-PAGE) and transferred to $0.22 \mu \mathrm{m}$ polyvinylidene fluoride (PVDF) membranes. Then blocking the membranes in $10 \%$ non-fat milk at room temperature for $1 \mathrm{~h}$, next incubating them with the primary antibodies, including anti-P-gp, anti-GAPDH overnight at $4^{\circ} \mathrm{C}$. The membranes were incubated with secondary antibody for $2 \mathrm{~h}$ at room temperature after 3 washes with Tris-buffered saline-Tween (TBST). The signal was visualized with an enhanced ECL detection kit (Beyotime, Shanghai, China).

shRNA. P-gp shRNAs were synthesized by Sangon Biotech (Shanghai, PR China) and cloned onto the pLVX plasmid. The sequences are shown in Table II. Non-silencing lentiviral shRNA vector was used as a negative control. To obtain stable P-gp knockdown cells, lentivirus supernatant was added to cells, which were then treated with $2 \mu \mathrm{g} / \mathrm{ml}$ puromycin for two weeks.

Mice. All mice were maintained in a specific pathogen-free (SPF) facility, and all animal experiments were conducted following the protocols approved by the ethics committee of the Shanghai Ninth
Table I. Sequences of the RT-qPCR primers.

\begin{tabular}{ll}
\hline P-gp-F & AATGCGACAGGAGATAGG \\
P-gp-R & TGTTGCCATTGACTGAAA \\
GAPDH-F & TCTGATTTGGTCGTATTGGG \\
GAPDH-R & GGAAGATGGTGATGGGATT \\
\hline
\end{tabular}

Table II. shRNA Sequences Targeting P-gp.

\begin{tabular}{ll}
\hline sh P-gp -1F & TTCCACTGTAATAATAGGC \\
sh P-gp -1R & GCCTATTATTACAGTGGAA \\
sh P-gp -2F & GCAGGAAATGAAGTTGAAT \\
sh P-gp -2R & ATTCAACTTCATTTCCTGC \\
\hline
\end{tabular}

People's Hospital. $2 \times 10^{6}$ HOS cells were suspended in sterile 100 $\mu \mathrm{l}$ PBS and injected subcutaneously into the flanks of 6-8-week-old male nude mice. After 7 days, the mice were divided into four groups randomly $(n=5)$ : Control; Dof. (dofequidar fumarate 200 $\mathrm{mg} / \mathrm{kg}$, p.o. $\mathrm{q} 3 \mathrm{~d} \times 6$ ); Cis (cisplatin $8 \mathrm{mg} / \mathrm{kg}$, i.p., q3d×6); Cis+Dof. (dofequidar fumarate $200 \mathrm{mg} / \mathrm{kg}$, p.o. and cisplatin $8 \mathrm{mg} / \mathrm{kg}$, i.p., $\mathrm{q} 3 \mathrm{~d} \times 6$ ). Administration of dofequidar fumarate was $30 \mathrm{~min}$ before the i.p. administration of cisplatin. Tumor sizes were measured as $0.5 \times$ length $\times$ width $^{2}$ every 7 days.

Statistical analysis. All experiments were conducted three times. The mean, standard error of mean (SEM) and $p$-values were analyzed by Excel (Microsoft) using the 2-tailed student's $t$-test. Differences were considered significant at $p<0.05 \quad\left({ }^{*} p<0.05\right.$, $* * p<0.01)$.

\section{Results}

$P$-gp was associated with cisplatin efficacy against $O S$. First, the relevance between $\mathrm{P}$-gp expression in patient specimens and cisplatin treatment efficacy was analyzed. According to the cisplatin sensitivity rate, the patients were divided into two groups: cisplatin sensitive group $(n=4)$ and cisplatin resistant group $(n=8)$. Approximately $70.51 \%$ of the OS cells responded to cisplatin treatment in the sensitive group while $6.85 \%$ responded in the resistant group. The expression profile of $\mathrm{P}$-gp analyzed by IHC indicated that P-gp expression was significantly higher in cisplatin -resistant specimens than in cisplatin sensitive specimens (Figure 1A and $\mathrm{B}$ ). Altogether, $\mathrm{P}-\mathrm{gp}$ was overexpressed in cisplatin resistant specimens compared to cisplatin sensitive ones.

OS cells with higher P-gp expression were more resistant to cisplatin. In order to verify the relationship between P-gp expression and cisplatin sensitivity in OS, the expression of P-gp in four OS cell lines was analyzed. HOS and U-2OS expressed higher levels of P-gp than MG-63 and Saos-2 did as assessed by RT-qPCR (Figure 2A) and western blot (Figure 

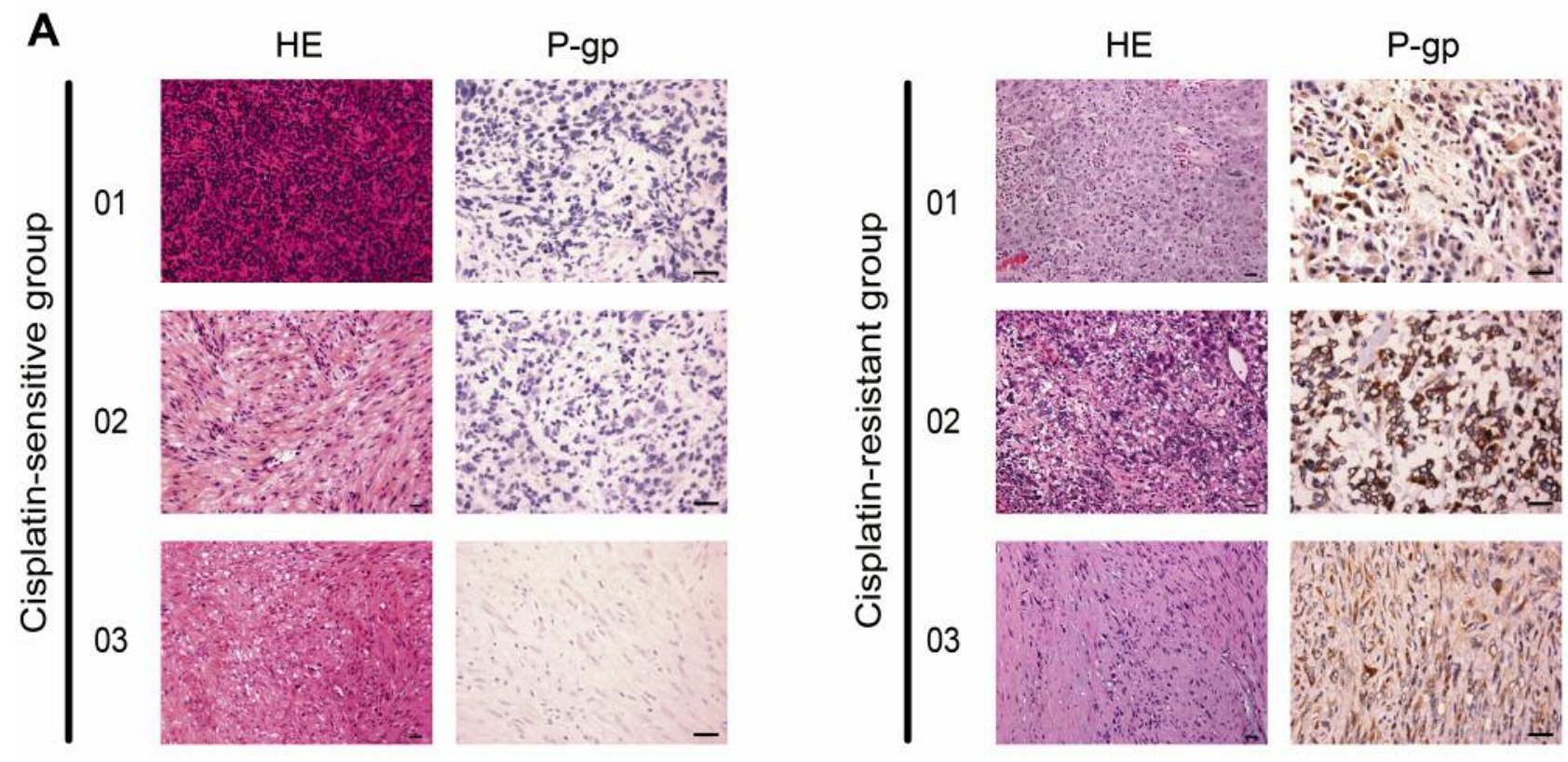

B

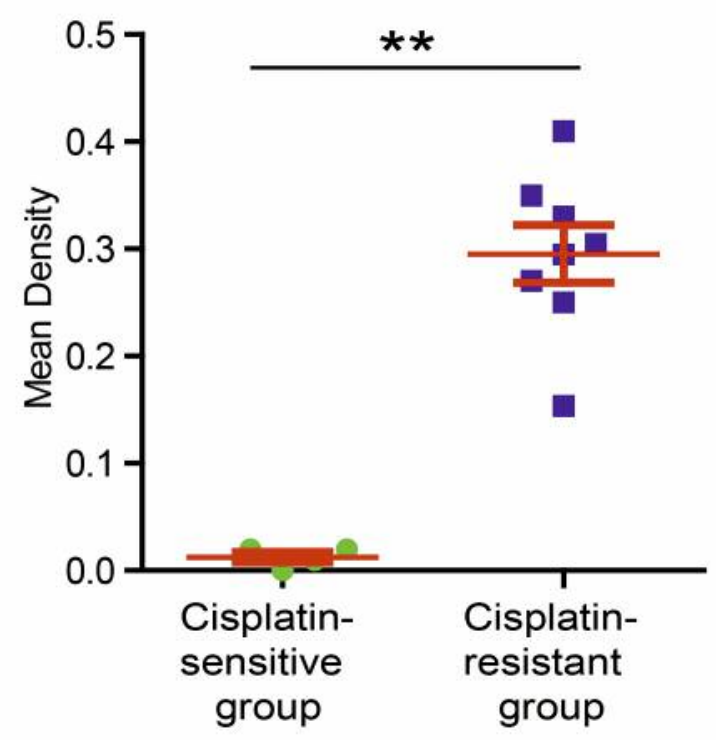

Figure 1. Expression of P-gp in OS patients. (A) Expression of P-gp was assessed in cisplatin-sensitive and -resistant OS patient specimens by immunohistochemical staining (IHC). (B) Quantification of the results from (A). **p<0.01. Scale bar $=100 \mu \mathrm{m}$.

2B). Furthermore, all cell lines showed a dose-dependent response to cisplatin treatment for $72 \mathrm{~h}$ (Figure 2C, D and E). HOS and U-2OS cells were more resistant to cisplatin than MG-63 and Saos-2 cells as measured by the CCK-8 assay (Figure 2C) and colony formation assay (Figure 2D and E). Together, our result indicated that OS cells with higher P-gp expression were more resistant to cisplatin.
Knockdown of P-gp could sensitize OS cells to cisplatin. To further test whether P-gp was involved in cisplatin resistance in OS, two shRNAs were designed to knockdown P-gp. The knockdown efficacy was verified by RT-qPCR (Figure 3A) and western blot (Figure 3B). P-gp-knockdown could significantly enhance cisplatin efficacy in both HOS and U-2OS cells as assessed by the CCK-8 assay (Figure 3C) and 
A

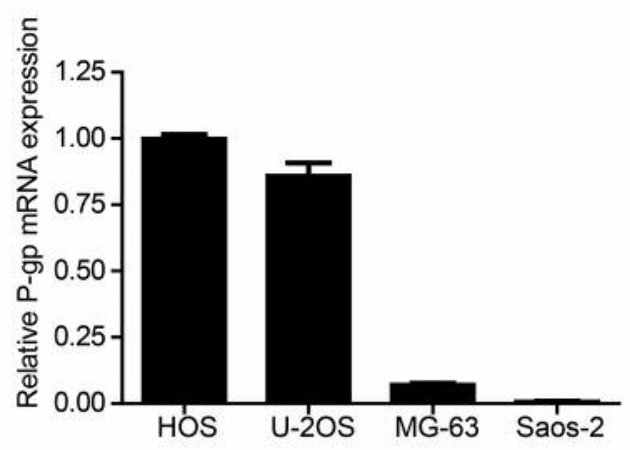

C

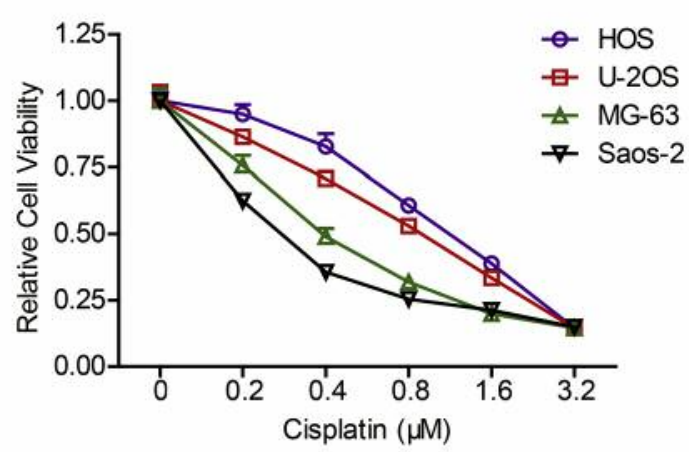

E

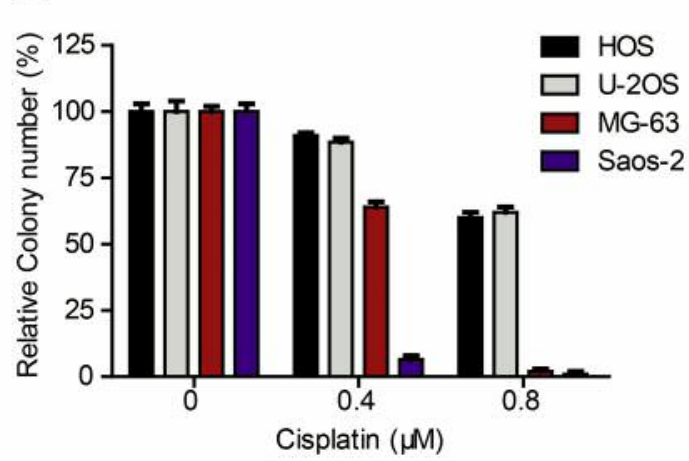

B

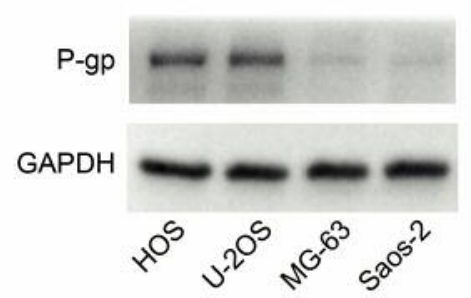

D
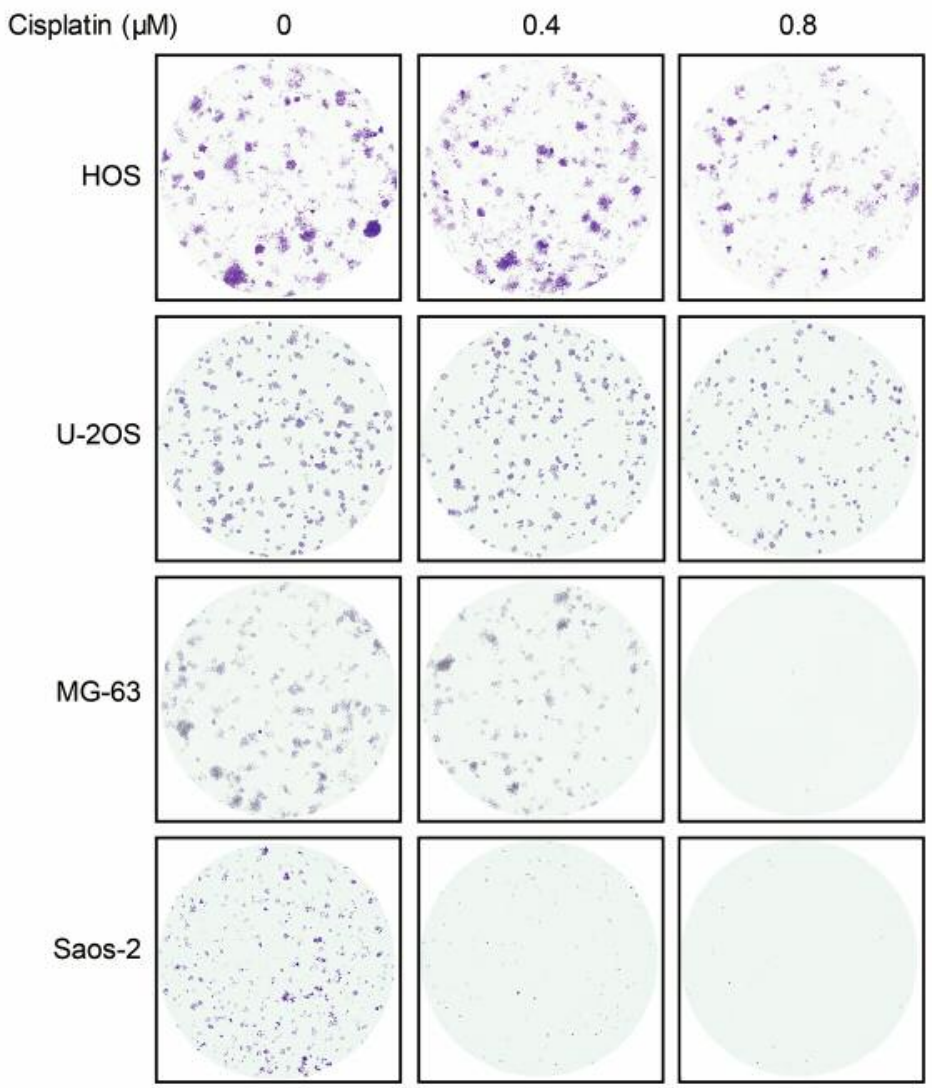

Figure 2. OS cells with higher P-gp were more resistant to cisplatin. P-gp expression in OS cells were analyzed by RT-qPCR (A) and western blot (B). Viability of OS cells exposed to increasing concentrations of cisplatin measured by CCK- 8 assay (C) and colony formation assay (D and E).

colony formation assay (Figure 3D). Collectively, knockdown of P-gp sensitized OS cells to cisplatin.

Dofequidar fumarate enhanced cisplatin efficacy on OS in vitro and in vivo. Additionally, dofequidar fumarate (Dof.) (17-20), an inhibitor of P-gp, was also used to further confirm the role of $\mathrm{P}$-gp in OS chemotherapy resistance. Dofequidar fumarate sensitized HOS and U-2OS to cisplatin, as determined by the CCK-8 (Figure 4A) and colony formation assays (Figure 4B). Moreover, dofequidar fumarate synergized with cisplatin in vivo. As shown, tumor weight and volume were much smaller in the Cis+Dof. group than in any of the other three groups (Figure 4C, $\mathrm{D}$ and E). Overall, these data indicated that inhibition of P-gp enhanced cisplatin efficacy in vitro and in vivo. 
A

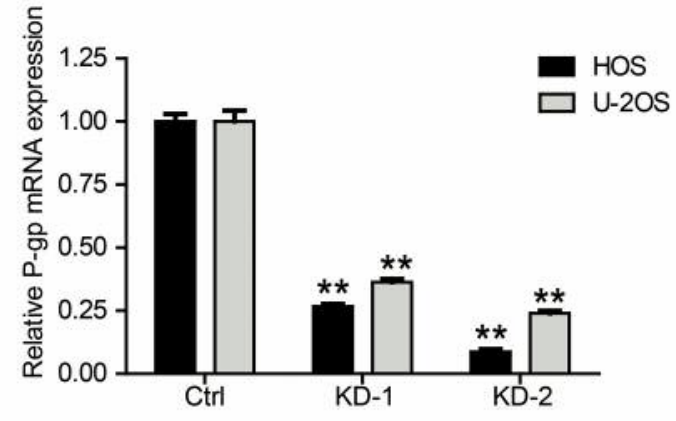

C

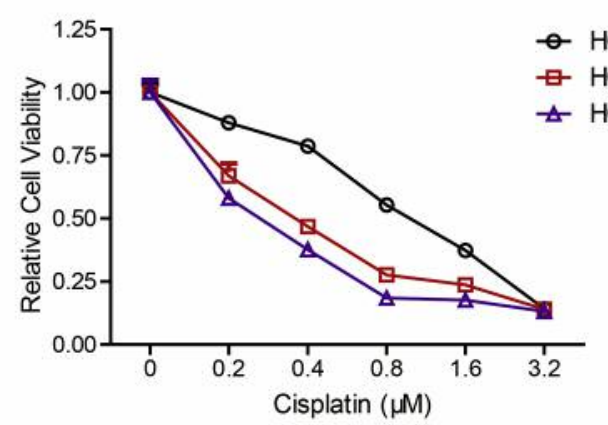

D

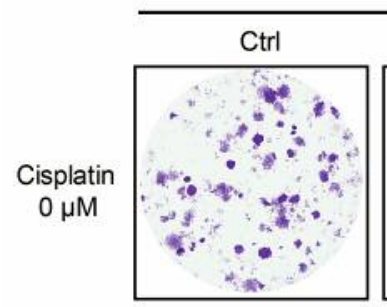

HOS
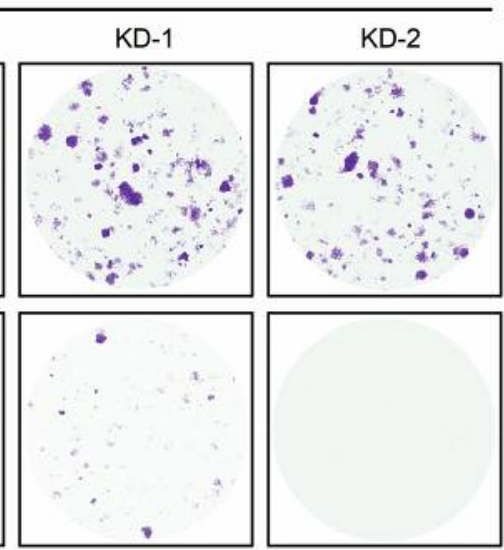

Cisplatin $0.8 \mu \mathrm{M}$
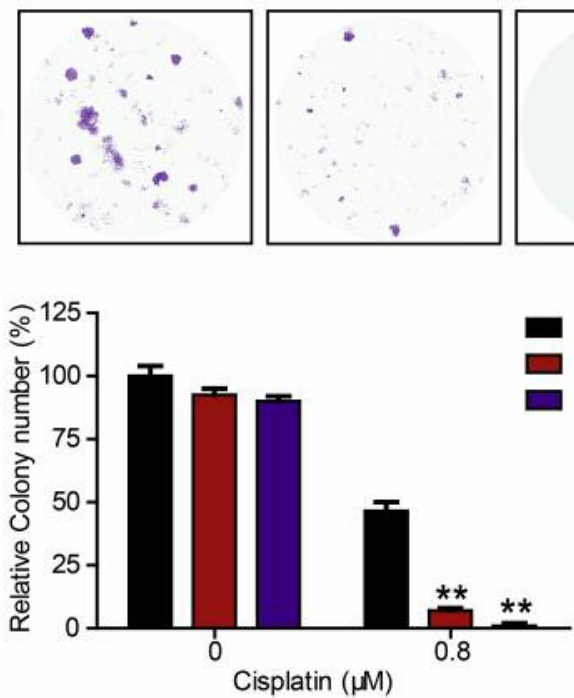

B
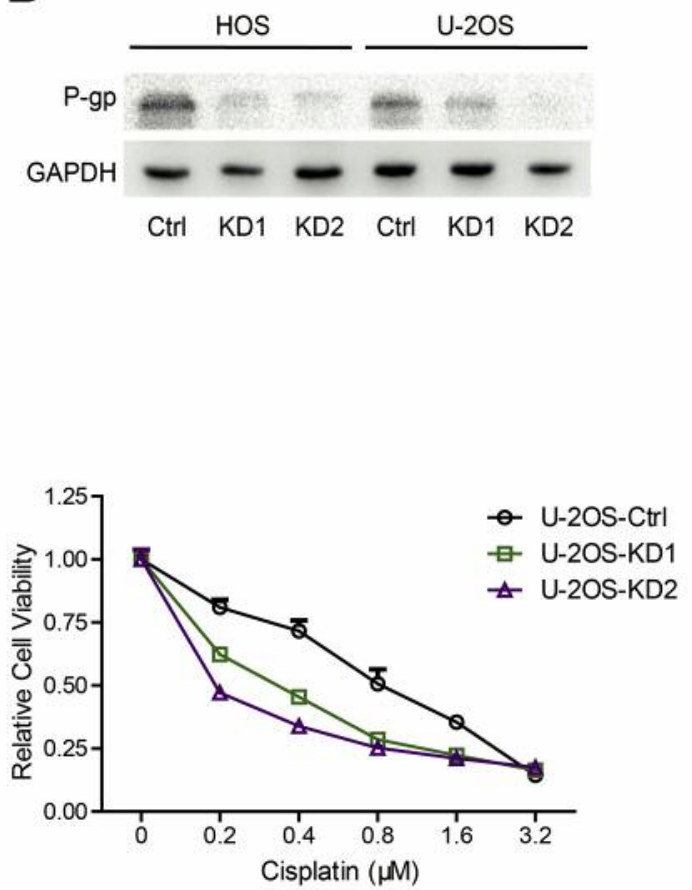

U-2OS
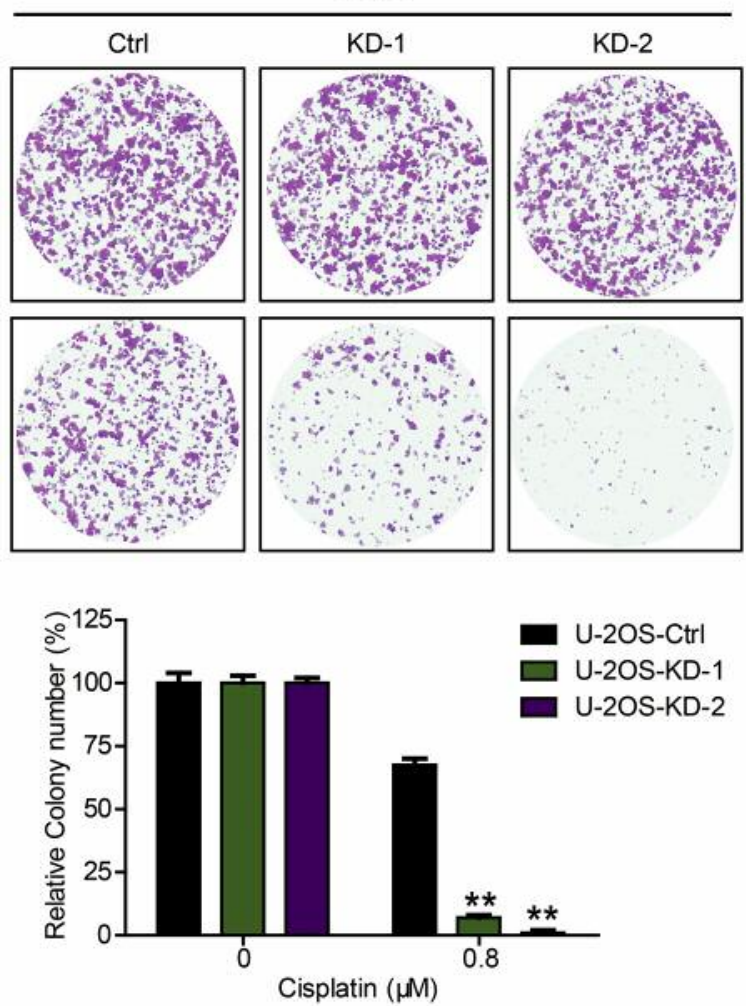

Figure 3. Knockdown of P-gp could sensitize OS cell to cisplatin. P-gp expression in HOS and U-2OS parental cells and P-gp knockdown cells $(K D-1$ and KD-2) determined by RT-qPCR (A) and western blot $(B)$. Viability of HOS and U-2OS parental cells and P-gp knockdown cells with the indicated treatment determined by the CCK-8 assay $(C)$ and colony formation assay $(D) . * * p<0.01$ compared with control. 
A

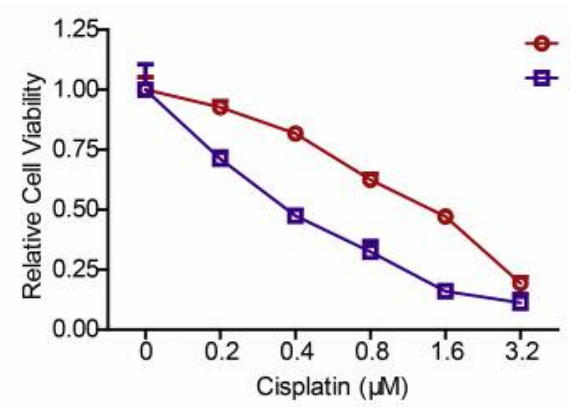

HOS-Ctrl

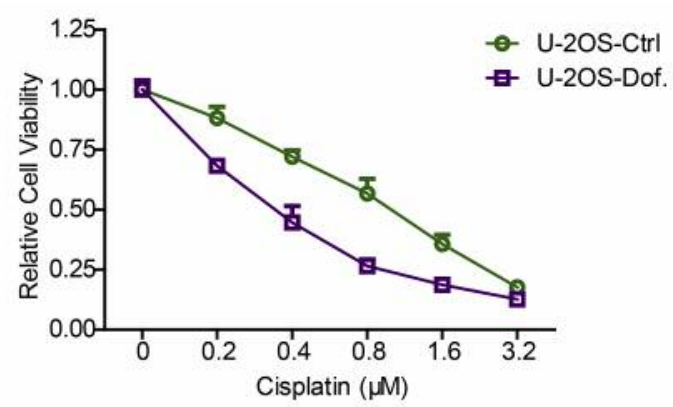

B

HOS

$\mathrm{U}-20 \mathrm{~S}$
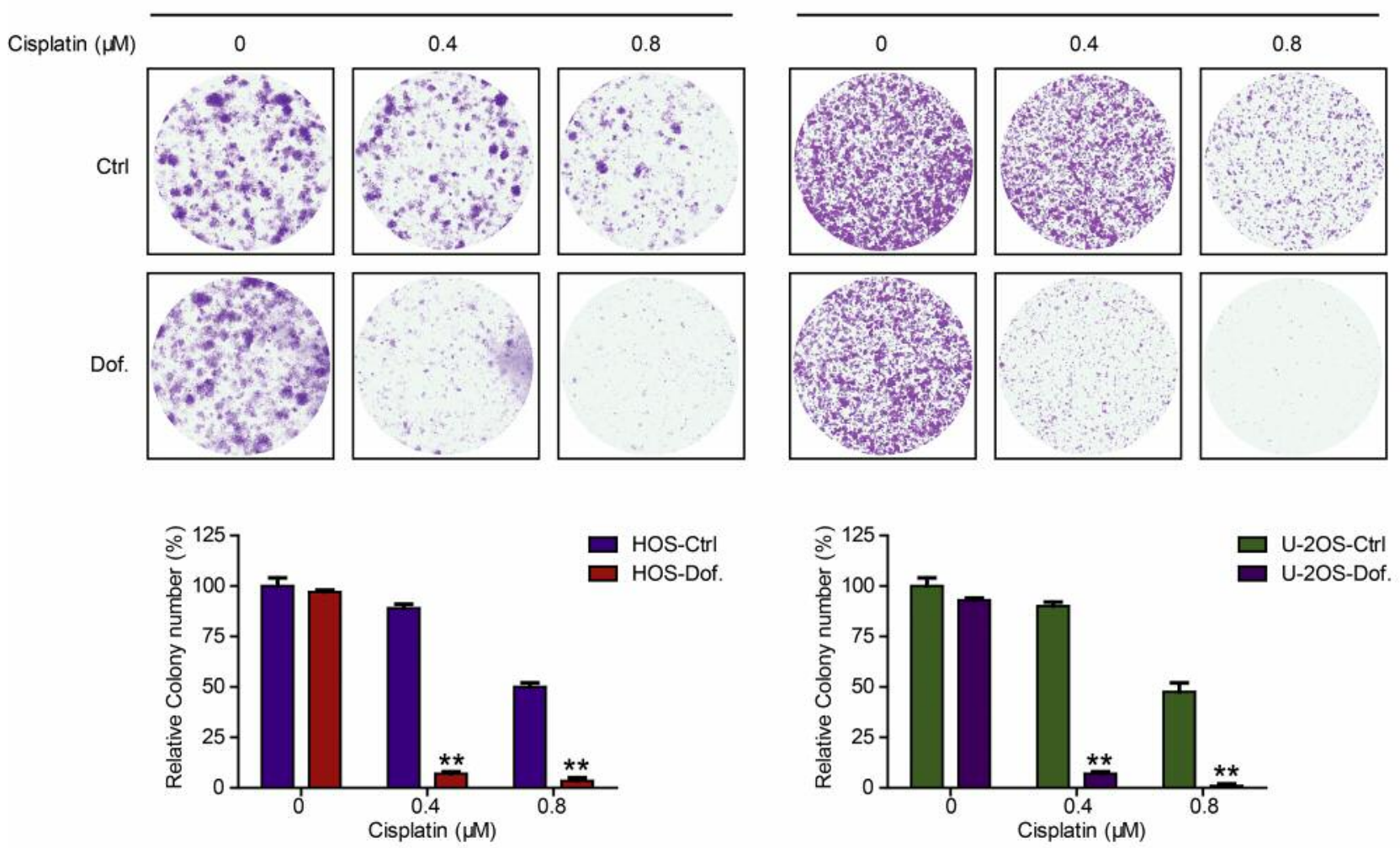

U-2OS-Ctrl

U-2OS-Dof.

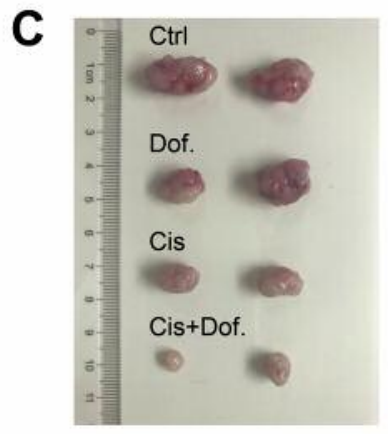

D

E
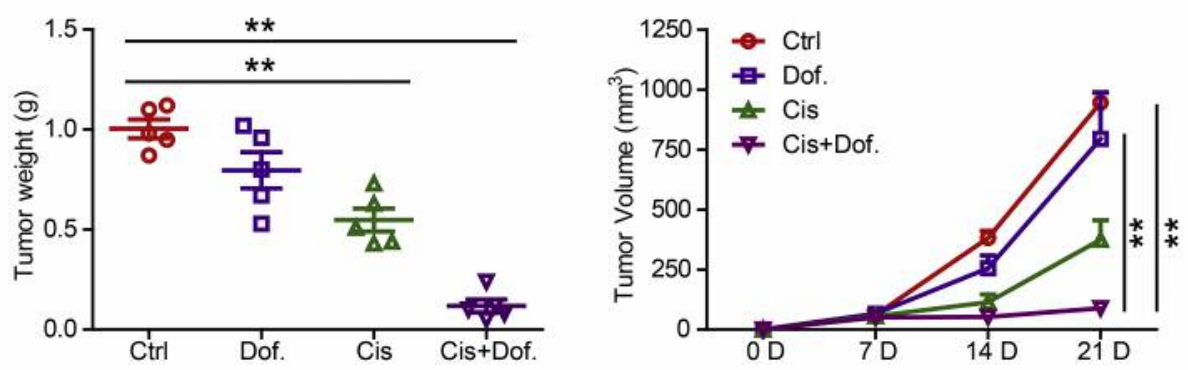

Figure 4. Dofequidar fumarate enhanced cisplatin efficacy against OS in vitro and in vivo. Viability of HOS and U-2OS cells exposed to increasing concentrations of cisplatin with or without dofequidar fumarate measured by CCK-8 assay (A) and colony formation assay (B). Representative images of tumors removed from mice $(C)$. Tumor weight $(D)$ and volume $(E)$ at the indicated times are shown in the diagram. $* * p<0.01$ compared with control. 


\section{Discussion}

OS is the most commonly primary malignancy of the bone diagnosed among children and adolescents (22-24). Despite the great advances in chemotherapy and surgery, the outcome of therapy for OS still remains unsatisfactory (25). Cisplatin is the most commonly used anti osteosarcoma drug due to its unique therapeutic advantages. However, cisplatin resistance is frequently reported, meaning that the enhancement of cisplatin sensitivity is imperative (26).

Several studies have reported that inhibition of P-gp reverses drug resistance in OS (27-30). In the current study, $\mathrm{P}$-gp was found to be overexpressed in cisplatin resistant OS specimens compared to cisplatin sensitive ones. OS cells with higher P-gp expression were more resistant to cisplatin. In addition, knockdown or inhibition of P-gp sensitized OS to cisplatin in vitro and in vivo.

Several drug resistance-related molecular mechanisms have been found in association with cisplatin resistance in OS. Kim et al. (31) revealed that GDNF receptor alpha 1 could overcome cisplatin resistance in OS by inhibiting AMP activated protein activated kinase-dependent autophagy. Zheng et al. (32) demonstrated that MAX dimerization protein 1 mediated hypoxia-induced cisplatin resistance in OS cells by suppressing the expression of phosphatase and tensin homolog. MicroRNAs have also been identified as novel modulators that regulate the effect of cisplatin in OS (33). Our study revealed an association of P-gp with cisplatin resistance in OS.

In conclusion, $\mathrm{P}$-gp mediated cisplatin resistance in OS, suggesting that $\mathrm{P}$-gp can be used as a potential therapeutic target for overcoming cisplatin resistance in OS.

\section{Conflicts of Interest}

The Authors declare no conflicts of interest regarding this study.

\section{Authors' Contributions}

Yongqiang Hao and Lei Wang designed the experiments, Chao He, Zhenyu Sun and Yuhang Jiang performed the experiments. Yuhang Jiang drafted the manuscript. Robert M. Hoffman and Zhijian Yang revised the manuscript. All Authors approved the final manuscript.

\section{Acknowledgements}

This study was supported by Grants from the Shanghai Ninth People's Hospital Lin+ Program (JYLJ012); the National Key R\&D Program of China (2016YFC1100600).

\section{References}

1 Bielack S, Carrle D and Jost L: Osteosarcoma: ESMO clinical recommendations for diagnosis, treatment and follow-up. Ann Oncol 19: ii94-96, 2008. PMID: 18456784. DOI: 10.1093/ annonc/mdn 102
2 Gillet JP and Gottesman MM: Mechanisms of multidrug resistance in cancer. Methods Mol Biol 596: 47-76, 2010. PMID: 19949920. DOI: 10.1007/978-1-60761-416-6_4

3 Gill J, Ahluwalia MK and Geller D: New targets and approaches in osteosarcoma. Pharmacol Ther 137: 89-99, 2013. PMID: 22983152. DOI: 10.1016/j.pharmthera.2012.09.003

4 Geller DS and Gorlick R: Osteosarcoma: a review of diagnosis, management, and treatment strategies. J Clin Adv Hematol Oncol 8: 705-718, 2010. PMID: 21317869.

5 Bielack SS, Marina N and Ferrari S: Osteosarcoma: the same old drugs or more? J Clin Oncol 26: 3102-3103, 2008. PMID: 18565904. DOI: $10.1200 / J C O .2008 .17 .1108$

6 Chou AJ, Geller DS and Gorlick R: Therapy for osteosarcoma: where do we go from here? Paediatr Drugs 10: 315-327, 2008. PMID: 18754698. DOI: 10.2165/00148581-200810050-00005

7 Susa M, Iyer AK and Ryu K: Inhibition of ABCB1 (MDR1) expression by an siRNA nanoparticulate delivery system to overcome drug resistance in osteosarcoma. PLoS One 5: e10764, 2010. PMID: 20520719. DOI: 10.1371/journal.pone.0010764

8 Chou AJ and Gorlick R: Chemotherapy resistance in osteosarcoma: current challenges and future directions. Expert Rev Anticancer Ther 6: 1075-1085, 2006. PMID: 16831079. DOI: $10.1586 / 14737140.6 .7 .1075$

9 Brambilla D, Zamboni S and Federici C: P-glycoprotein binds to ezrin at amino acid residues 149-242 in the FERM domain and plays a key role in the multidrug resistance of human osteosarcoma. Int J Cancer 130: 2824-2834, 2012. PMID: 21780101. DOI: $10.1002 /$ ijc. 26285

10 Kobayashi E, Iyer AK and Hornicek FJ: Lipid-functionalized dextran nanosystems to overcome multidrug resistance in cancer: a pilot study. Clin Orthop Relat Res 471: 915-925, 2013. PMID: 23011844. DOI: 10.1007/s11999-012-2610-2

11 Juliano RL and Ling V: A surface glycoprotein modulating drug permeability in Chinese hamster ovary cell mutants. Biochim Biophys Acta 455: 152-162, 1976. PMID: 990323.

12 Gottesman MM, Fojo T and Bates SE: Multidrug resistance in cancer: role of ATP-dependent transporters[J]. Nat Rev Cancer 2: 48-58, 2002. PMID: 11902585. DOI: $10.1038 / \mathrm{nrc} 706$

13 Krishna R and Mayer LD: Multidrug resistance (MDR) in cancer - Mechanisms, reversal using modulators of MDR and the role of MDR modulators in influencing the pharmacokinetics of anticancer drugs. Eur J Pharm Sci 11: 265-283, 2000. PMID: 11033070.

14 Goldstein LJ, Galski H and Fojo A: Expression of a multidrug resistance gene in human cancers. J Natl Cancer Inst 81: 116124, 1989. PMID: 2562856.

$15 \mathrm{He}$ C, Sun J and Liu C: Elevated H3K27me3 levels sensitize osteosarcoma to cisplatin. Clin Epigenetics 11: 8, 2019. PMID: 30651137. DOI: 10.1186/s13148-018-0605-x

$16 \mathrm{He} \mathrm{C}, \mathrm{Wu} \mathrm{T}$ and Hao Y: Anlotinib induces hepatocellular carcinoma apoptosis and inhibits proliferation via Erk and Akt pathway. Biochem Biophys Res Commun 503: 3093-3099, 2018. PMID: 30146257. DOI:10.1016/j.bbrc.2018.08.098

17 Nokihara H, Yano S and Nishioka Y: A new quinoline derivative MS-209 reverses multidrug resistance and inhibits multiorgan metastases by P-glycoprotein-expressing human small cell lung cancer cells. Jpn J Cancer Res 92: 785-792, 2001. PMID: 11473730.

18 Nakanishi O, Baba M and Saito A: Potentiation of the antitumor activity by a novel quinoline compound, MS-209, in multidrugresistant solid tumor cell lines. Oncol Res 9: 61-69, 1997. PMID: 9167187. 
19 Kubo T, Ashihara T and Tsubouchi T: Significance of integrated in silico transmural ventricular wedge preparation models of human non-failing and failing hearts for safety evaluation of drug candidates. J Pharmacol Toxicol Methods 83: 30-41, 2017. PMID: 27546811. DOI: 10.1016/j.vascn.2016.08.007

20 Katayama R, Koike S and Sato S: Dofequidar fumarate sensitizes cancer stem-like side population cells to chemotherapeutic drugs by inhibiting ABCG2/BCRP-mediated drug export. Cancer Sci 100: 2060-2068, 2009. PMID: 19673889. DOI: 10.1111/j.1349-7006.2009.01288.x

$21 \mathrm{He}$ C, Liu C and Wang L: Histone methyltransferase NSD2 regulates apoptosis and chemosensitivity in osteosarcoma. Cell Death Dis 10: 65, 2019. PMID: 30683853. DOI: 10.1038/s 41419-019-1347-1

22 Sweetnam R: Osteosarcoma. Br J Hospital Med 28: 112-121, 1982.

23 Dorfman HD and Czerniak B: Bone cancers. Cancer 75: 203 210, 1995. PMID: 8000997.

24 Damron TA, Ward WG and Stewart A: Osteosarcoma, chondrosarcoma, and Ewing's sarcoma: National cancer data base report. Clin Orthop Relat Res 459: 40-47, 2007. PMID: 17414166. DOI: $10.1097 / B L O .0 b 013 e 318059 b 8 c 9$

25 Takeda S, Iwai A and Nakashima M: LKB1 is crucial for TRAIL-mediated apoptosis induction in osteosarcoma. Anticancer Res 27: 761-768, 2007. PMID: 17465200.

26 Yang J, Guo W, Wang L, Yu L, Mei H, Fang S, Ji P, Liu Y, Liu $\mathrm{G}$ and Song Q: Cisplatin resistant osteosarcoma cells possess cancer stem cell properties in a mouse model. Oncol Lett 12: 2599 2605, 2016. PMID: 27698833. DOI: 10.3892/ol.2016.4956

27 Ye S, Zhang J and Shen J: NVP-TAE684 reverses multidrug resistance (MDR) in human osteosarcoma by inhibiting Pglycoprotein (PGP1) function. Br J Pharmacol 173: 613-626, 2016. PMID: 26603906. DOI:10.1111/bph.13395
28 Yang X, Yang $\mathrm{P}$ and Shen J: Prevention of multidrug resistance (MDR) in osteosarcoma by NSC23925. Br J Cancer 110: 28962904, 2014. PMID: 24853187. DOI: 10.1038/bjc.2014.254

29 Fanelli M, Hattinger CM and Vella S: Targeting ABCB1 and ABCC1 with their specific inhibitor CBT-1(R) can overcome drug resistance in osteosarcoma. Curr Cancer Drug Targets 16: 261-274, 2016. PMID: 26548759.

30 Perez J, Bardin C and Rigal C: Anti-MDR1 siRNA restores chemosensitivity in chemoresistant breast carcinoma and osteosarcoma cell lines. Anticancer Res 31: 2813-2820, 2011. PMID: 21868524.

31 Kim M, Jung JY, Choi S, Lee H, Morales LD, Koh JT, Kim SH, Choi YD, Choi $\mathrm{C}$ and Slaga TJ: GFRA1 promotes cisplatin induced chemoresistance in osteosarcoma by inducing autophagy. Autophagy 13: 149 168, 2017. PMID: 27754745. DOI: $10.1080 / 15548627.2016 .1239676$

32 Zheng D, Wu W, Dong N, Jiang X, Xu J, Zhan X, Zhang Z and $\mathrm{Hu} \mathrm{Z}$ : Mxd1 mediates hypoxia induced cisplatin resistance in osteosarcoma cells by repression of the PTEN tumor suppressor gene. Mol Carcinog 56: 2234 2244, 2017. PMID: 28543796. DOI: $10.1002 / \mathrm{mc} .22676$

33 Zou Y, Yang J, Wu J, Luo C and Huang Y: miR 133b induces chemoresistance of osteosarcoma cells to cisplatin treatment by promoting cell death, migration and invasion. Oncol Lett 15: 1097-1102, 2018. PMID: 29399170. DOI:10.3892/ol.2017.7432

Received February 18, 2019

Revised March 5, 2019

Accepted March 15, 2019 